\title{
O sistema de medicação nos hospitais e sua avaliação por um grupo de profissionais
}

\author{
MEDICATION SYSTEMS IN HOSPITALS AND THEIR EVALUATION BY PROFESSIONAL GROUPS \\ EL SISTEMA DE MEDICACIÓN EN LOS HOSPITALES Y SU EVALUACIÓN \\ POR UN GRUPO DE PROFESIONALES
}

Silvia Helena De Bortoli Cassiani ${ }^{1}$, Thalyta Cardoso Alux Teixeira ${ }^{2}$,
Simone Perufo Opitz ${ }^{3}$, Josilene Cristina Linhares ${ }^{4}$

1 Enfermeira. Professora Associada do Departamento de Enfermagem Geral e Especializada da Escola de Enfermagem de Ribeirão Preto da Universidade de São Paulo (EERP-USP).

2 Acadêmica do $6^{\circ}$ semestre da EERP-USP. Bolsista de Iniciação Científica do CNPq.

thalytacat@yahoo.com.br

3 Enfermeira. Mestre e

doutoranda do Programa de

Enfermagem Fundamental

da EERP-USP. Docente

da Universidade Federal do Acre.

4 Acadêmica do $4^{\circ}$ semestre da EERP-USP. Bolsista de Iniciação Científica do $\mathrm{CNPq}$

\section{RESUMO}

O objetivo do estudo foi analisar os sistemas de medicação, em hospitais, a partir da opinião de 107 profissionais. Em relação às prescrições médicas nas instituições, 74,8\% eram manuais e $50,4 \%$ dos sistemas de distribuição de medicamentos eram doses individualizadas. Quanto às causas dos erros na medicação, $91 \%$ estavam associadas ao profissional. Para $61,7 \%$, o sistema estava adequado, mas apresentando falhas. Poucos profissionais sugeriram modificações que favoreceriam seu trabalho. Conclui-se que ainda persiste a cultura de responsabilizar o profissional pelo erro e, também, a prática de punição, sem modificação substancial da causa que levou ao erro.

\section{DESCRITORES}

Sistemas de medicação no hospital.

Erros de medicação.

Prescrição de medicamentos.

\begin{abstract}
This study was aimed at analyzing the medication systems in hospitals based on the opinions of 107 professionals. With regard to medical prescriptions in the institutions, 74.8 percent were handwritten and 50.4 percent of the medication distribution systems were in individualized doses. Concerning the causes of medication errors, 91 percent were associated with the professionals. For 61.7 percent, the system was adequate, but had shortcomings. Few professionals suggested changes that would favor their work. The conclusion is that the culture of attributing responsibility to the professional for the error, as well as the practice of punishment without a substantial change of the cause that led to it, persists.
\end{abstract}

\section{KEY WORDS}

Medication systems, Hospital. Medication errors.

Prescriptions, drug.

\section{RESUMEN}

El objetivo de este estudio fue analizar los sistemas de medicación en los hospitales, a partir de la opinión de un grupo de 107 profesionales. Con relación a las prescripciones médicas en las instituciones, un $74,8 \%$ eran manuales y un 50,4\% de los sistemas de distribución de medicamentos eran dosis individualizadas. En lo que se refiere a las causas de los errores en la medicación, un 91\% estaban asociadas al profesional. Para un $61,7 \%$ el sistema era adecuado, sin embargo presentaba fallas. Pocos profesionales sugirieron algunas modificaciones que favorezcan su trabajo. Se llegó a la conclusión de que aún persiste la costumbre de responsabilizar al profesional por el error y, también, la práctica de punición, sin modificar substancialmente la causa que llevó a cometer el error.

\section{DESCRIPTORES}

Sistemas de medicación en hospital.

Errores de medicación. Prescripción de medicamentos. 


\section{INTRODUÇÃO}

O sistema de medicação é constituído de várias etapas que vão desde a prescrição e distribuição até a ação de administrar o medicamento ao cliente.

Todas essas etapas dependem de vários profissionais da área de saúde e estão diretamente interligadas. Assim, a prescrição é de responsabilidade do médico, a dispensação e distribuição do medicamento estão sob a responsabilidade do farmacêutico e a administração bem como o monitoramento das reações do cliente são responsabilidade do enfermeiro.

O Instituto de Medicina Americano indicou que 44.000 a 98.000 americanos morrem a cada ano por erros na medicação. Entre 1983 e 1993, as mortes relacionadas à medicação cresceram na ordem de $257 \%{ }^{(1)}$. Estima-se que em cada dez clientes admitidos no hospital, um está em risco para erro potencial ou efetivo na medicação ${ }^{(2)}$.

Algumas definições importantes para o entendimento dessa questão precisam ser destacadas. Eventos (ou reações) adversos são definidos como qualquer injúria devido à medicação. Esses eventos podem ser preveníveis (ex: dose errada) ou não preveníveis (vermelhidão devido a um antibiótico). Erro na medicação é definido como qualquer erro que ocorreu durante o processo de medicação, nas fases de prescrição, dispensação, administração e monitoramento. Eventos adversos potenciais são eventos nos quais um erro na medicação ocorreu, mas não causou injúria por alguma razão (ex: o erro foi interceptado antes do cliente ser afetado ou o cliente recebeu uma dose errada, mas nenhuma lesão ocorreu). Todos os eventos potenciais são erros na medicação $0^{(3)}$.

Os erros de medicação podem ocorrer em qualquer etapa do sistema, podendo acarretar injúrias ao cliente devido aos efeitos adversos da medicação. Alguns estudos afirmam que erros na medicação ocorrem freqüentemente na prescrição e administração do medicamento ${ }^{(4-5)}$.

Assim, a supervisão junto aos processos de prescrição, preparação e administração da droga são importantes meios para a redução dos eventos $\operatorname{adversos}^{(6)}$.

Várias estratégias têm sido tomadas para reduzir os erros na prescrição médica, como a padronização dos processos, uso da prescrição eletrônica, a educação do profissional que prescreve e uma expansão da ação do farmacêutico para uma melhor integração à equipe de saúde $\mathrm{e}^{(7)}$.
A ação multidisciplinar dos profissionais e a participação de farmacêuticos são uma estratégia importante e fundamental, pois esse profissional atua na prevenção e interceptação do erro, prevenindo os efeitos adversos das medicações ${ }^{(8)}$.

As principais recomendações apresentadas pela American Society of Hospital Pharmacists (ASHP) para evitar erros na medicação são: informatização do sistema (prescrição, dispensação, distribuição do medicamento); uso do código de barras nos processos de medicação e na identificação do cliente; sistemas de monitoramento e relatórios de eventos adversos; uso da distribuição por dose unitária; sistema para preparação de medicação intravenosa realizada pela farmácia; profissionais da farmácia atuando junto com médicos e enfermeiros; revisão das prescrições por farmacêuticos e a resolução das dúvidas sobre os medicamentos ${ }^{(9)}$.

O estudo realizado em 36 instituições hospitalares americanas verificou que $19 \%$ das doses administradas estavam erradas, sendo que as categorias mais freqüentes de erros relacionavam-se ao horário errado (43\%), omissão das doses (30\%), dose errada (17\%) e medicamento não prescrito $(4 \%)^{(10)}$.

A administração de medicamentos é de responsabilidade da equipe de enfermagem, sobretudo em ambiente hospitalar, devendo ao enfermeiro a supervisão e a orientação da equipe quanto aos aspectos e princípios relacionados ao uso dos fármacos prescritos ${ }^{(11)}$.

A equipe de enfermagem atua na parte final do processo de medicação, podendo prevenir os erros que porventura aconteceram em outra etapa e sendo normalmente responsabilizada por esse erro.

Os estudos têm demonstrado que os erros são causados por falhas no sistema de medicação, ao invés de somente falhas nas pessoas. Entretanto, ainda, persiste a cultura de punir o profissional quando detectado o erro de medicação. Desse modo, os profissionais deparam-se freqüentemente com ações disciplinares, humilhações, demissão e repercussão legal, quando os erros de medicação são relatados ${ }^{(12)}$.

O Instituto Americano de Medicina indica que um meio de aprender com os erros e interferir no sistema é estabelecer um instrumento eficiente de relatos voluntários e anônimos e que este é útil em identificar os tipos de erros que ocorrem de forma usual ou que são problemas emergentes ${ }^{(1)}$.
O sistema de medicação nos hospitais e sua avaliação por um grupo de profissionais 


\section{OBJETIVO}

O objetivo deste estudo foi analisar os sistemas de medicação em hospitais, a partir da opinião dos profissionais que atuavam nessas instituições.

\section{METODOLOGIA}

\section{Tipo de estudo}

Trata-se de um estudo exploratório-descritivo, a partir da opinião de profissionais, sobre o sistema de medicação das instituições onde eles atuavam.

\section{População e amostra}

A amostra acidental constitui-se de 107 participantes, sendo 95 enfermeiros, 6 farmacêuticos, 4 técnicos de enfermagem e 2 auxiliares de enfermagem.

\section{Procedimentos de coleta de dados}

A coleta de dados teve início após a aprovação do projeto pelo Comitê de Ética em Pesquisa da Escola de Enfermagem de Ribeirão Preto - USP.

Elaborou-se um questionário composto por questões abertas e fechadas, as quais identifica- vam o profissional, o sistema de distribuição de medicamento, as causas e atitudes tomadas pela instituição frente ao erro na medicação, a opinião dos profissionais sobre as atitudes e o sistema e as sugestões dos participantes para modificações na instituição.

Os questionários foram enviados pelo correio ou entregues pessoalmente para os profissionais que participaram de um evento científico. Todos foram orientados sobre o estudo e assinaram o termo de consentimento informado.

\section{RESULTADOS}

A Tabela 1 indica que dos 107 participantes, $88,8 \%$ eram enfermeiros, $5,6 \%$ farmacêuticos, $3,7 \%$ técnicos de enfermagem e 1,9\% auxiliares de enfermagem. Com relação aos profissionais de enfermagem, $87,1 \%$ atuavam diretamente na assistência ao cliente ou administrativamente, $11,9 \%$ atuavam no ensino da profissão e $1 \%$ era enfermeiro que atuava em órgão fiscalizador da profissão.

Desses profissionais, $61,7 \%$ atuavam na instituição a menos de 10 anos, $26,2 \%$ atuavam a mais de 10 anos e menos que 20 anos e $12,1 \%$ atuavam a mais de 20 anos e menos de 30 anos.

Tabela 1- Distribuição dos participantes do estudo segundo atividade profissional, área profissional da enfermagem e tempo de atuação no hospital. (Ribeirão Preto, 2003)

\begin{tabular}{llc}
\hline & & $\mathbf{N}(\%)$ \\
\hline \multirow{2}{*}{ Atividade profissional } & Enfermeiro & $95(88,8)$ \\
& Farmacêutico & $6(5,6)$ \\
& Técnico de enfermagem & $4(3,7)$ \\
& Auxiliar de enfermagem & $2(1,9)$ \\
& Total & $107(100)$ \\
Área profissional da enfermagem & Assistencial ou administrativa & $88(87,1)$ \\
& Ensino & $12(11,9)$ \\
& Órgão profissional & $1(1)$ \\
& & \\
Tempo de atuação & Total & $101(100)$ \\
& $0 \mid-5$ & $32(29,9)$ \\
& $5 \mid-10$ & $34(31,8)$ \\
& $10 \mid-20$ & $28(26,2)$ \\
& $20 \mid-30$ & $13(12,1)$ \\
\hline
\end{tabular}

A Tabela 2 apresenta o tipo de prescrição médica e a relação dos medicamentos, segundo o nome comercial ou genérico. Observou-se que, em 74,8\% (80) das respostas, houve a informação de que nas instituições a prescrição médica era manual, sendo $43,9 \%$ (47) redigidas com o nome genérico e/ou comercial, $21,5 \%$ (23) com o nome comercial e $9,4 \%$
(10) com o nome genérico. Em 18,7\% (20), as prescrições eram eletrônicas, sendo 9,4\% (10) escritas com o nome genérico e/ou comercial, 8,4\% (9) com o nome genérico e $0,9 \%$ (1) com o nome comercial. Em 6,5\% (7) eram mistas, ou seja, manual e eletrônica. Dessas, 5,6\% (6) eram escritas com o nome genérico e/ou comercial e $0,9 \%$ com o nome genérico. 
Tabela 2- O tipo de prescrição médica segundo as instituições dos participantes do estudo. (Ribeirão Preto, 2003)

\begin{tabular}{llrr}
\hline \multicolumn{1}{c}{ Tipo de prescrição médica } & \multicolumn{1}{c}{ Nome } & $\mathbf{N}^{\mathbf{o}}$ & $\%$ \\
\hline \multirow{2}{*}{ Manual } & Genérico e/ou comercial & 47 & 43,9 \\
& Comercial & 23 & 21,5 \\
\multirow{2}{*}{ Eletrônica } & Genérico & 10 & 9,4 \\
& Nome genérico e/ou comercial & 10 & 9,4 \\
\multirow{2}{*}{ Mista(eletrônica e/ou manual) } & Nome genérico & 9 & 8,4 \\
& Nome comercial & 1 & 0,9 \\
& Nome genérico e/ou comercial & 6 & 5,6 \\
Total & Nome genérico & 1 & 0,9 \\
\hline
\end{tabular}

Em relação ao sistema de distribuição de medicamentos nas instituições, ele está descrito na Tabela 3. Dos 107 participantes, 50,4\% (54) relataram

que o tipo de distribuição era por dose individualizada; $18,7 \%$ (20) por dose unitária, $14,9 \%$ (16) por farmácia-satélite, entre outras.

Tabela 3- O tipo de sistema de distribuição de medicamentos nas instituições dos participantes do estudo. (Ribeirão Preto, 2003)

\begin{tabular}{lcc}
\hline Tipo de sistema & $\mathbf{N}^{\mathbf{0}}$ & \% \\
\hline Dose individualizada & 54 & 50,4 \\
Dose unitária & 20 & 18,7 \\
Farmácia-satélite & 16 & 14,9 \\
Mista(unitária e individualizada) & 5 & 4,7 \\
Coletiva & 2 & 1,9 \\
Farmácia centralizada & 2 & 1,9 \\
Distribuição por áreas & 1 & 0,9 \\
Outras & 5 & 4,7 \\
Não responderam & 2 & 1,9 \\
\hline Total & 107 & 100 \\
\hline
\end{tabular}

Quanto às causas dos erros de medicação citadas pelos participantes do estudo, nota-se que para $91 \%$ (232) dos participantes as causas estavam associadas ao profissional, destas $31,8 \%(81)$ relacionaram à falta de atenção, $17,2 \%$ (44), à falta de treinamento, $16,9 \%$ (43), à falta de conhecimen- to e $16,1 \%$ (41), à falta de comunicação entre os profissionais (enfermeiros, médicos e farmacêuticos). No restante das causas, $9 \%$ (23) foram associadas ao sistema sendo referido principalmente por eles a não-implantação da dose unitária e problemas na prescrição médica (Tabela 4).

Tabela 4- Distribuição das causas dos erros de medicação segundo os relatos dos participantes do estudo. (Ribeirão Preto, 2003)

\begin{tabular}{llcc}
\hline & Causas dos erros de medicação & $\mathbf{N}^{\mathbf{0}}$ & $\%$ \\
\hline \multirow{2}{*}{ Associadas ao profissional } & Falta de atenção & 81 & 31,8 \\
& Falta de treinamento & 44 & 17,2 \\
& Falta de conhecimento & 43 & 16,9 \\
& Falta de comunicação & 41 & 16,1 \\
& Negligência & 18 & 7,0 \\
& Excesso de trabalho & 5 & 2 \\
Associadas ao sistema & Não-implantação da dose unitária & 232 & 91 \\
& Problemas na prescrição médica & 16 & 6,3 \\
& Falha do sistema $\quad$ Subtotal & 6 & 2,3 \\
& & 1 & 0,4 \\
\hline \multirow{2}{*}{ Total } & & 23 & 9 \\
\hline
\end{tabular}


Silvia H. De Bortoli Cassiani Thalyta Cardoso A. Teixeira Simone Perufo Opitz Josilene Cristina Linhares

No que refere às atitudes tomadas pelas instituições frente ao erro de medicação, a Tabela 5 indica que $88,9 \%$ das atitudes foram tomadas em relação ao próprio profissional, sendo reunião com o profissional $(32,7 \%)$, relato da ocorrência do erro $(26,6 \%)$, treinamento e orientações ao profissional $(26,6 \%)$.

Tabela 5 - Distribuição dos tipos de atitudes tomadas pelas instituições frente ao erro de medicação. (Ribeirão Preto, 2003)

\begin{tabular}{llcc}
\hline & Tipos de atitudes & No & $\%$ \\
\hline Com o profissional & Reunião & 86 & 32,7 \\
& Relato de ocorrência & 70 & 26,6 \\
& Treinamento e orientação & 70 & 26,6 \\
& Advertência, suspensão ou demissão & 6 & 2,3 \\
& Análise do processo de trabalho e & 2 & 0,7 \\
Com o cliente & mudança de escala & 27 & 10,3 \\
Com a equipe & Comunica cliente ou família & 1 & 0,4 \\
Não há constatação & Comunica médico & 1 & 0,4 \\
\hline \multirow{2}{*}{ Total } & & $263^{*}$ & 100 \\
\hline
\end{tabular}

* Houve vários tipos de atitudes tomadas pela instituição e relatas pelo mesmo profissional.

Quando questionados sobre as atitudes tomadas pela instituição frente ao erro na medicação 75,7\% (81) concordaram, 18,7\% (20) discordaram com as atitudes adotadas pela sua instituição e 5,6\% (6) não responderam. Dos que concordaram, suas justificativas foram agrupadas. Assim, $31,8 \%$ (34) justificaram em relação a aspectos do funcionário afirmando que os profissionais devem responder por seus atos e atitudes e citaram, também, a falta de atenção, a falta de conhecimento e pouca concentração como algumas das causas que levaram aos erros na medicação; 7,5\% (8) responderam em relação a aspectos do cliente, pois os erros podem acarretar problemas ou riscos ao cliente; 3,7\% (4) justificaram em relação a aspectos do sistema, comentando que ele é falho, e que têm que ser tomadas atitudes para obter uma melhor qualidade do sistema; 27,2\% (29) responderam que são vários fatores envolvidos no erro e com isso devem ser analisados.

Dos participantes que discordaram 18,7\%, relataram que existem vários fatores envolvidos no erro na medicação e por isso não devem ser tomadas atitudes em relação a apenas um aspecto, como por exemplo, o profissional. Segundo esses participantes, deve-se investir em atualizações periódicas para todos os profissionais e não somente para aquele que cometeu o erro. Eles afirmaram, também, que o relato de ocorrência do erro deve ocorrer na instituição, para que se possa analisar a causa do erro.

A Tabela 6 apresenta as sugestões citadas pelos participantes para modificações nas instituições. Observou-se que a maioria foram sugestões para implantação ou modificações na instituição.

Destacamos a implantação da dose unitária $(15,9 \%)$, reserva de medicamentos mais solicitados nos setores $(8 \%)$, dose individual $(7,1 \%)$ e informatização do sistema de medicação $(5,3 \%)$ como as sugestões mais relatadas pelos participantes.

Para modificações na instituição, 7,1\% das sugestões foram em relação às atitudes adotadas pela instituição com o profissional, sendo enfatizado por esses participantes a adoção de atividades com a finalidade de evitar erros de medicação e não com o objetivo de punir o profissional, quando constatado o erro na medicação; 3,5\% foram para modificar a forma de prescrever medicações; $3,5 \%$ foram em relação à distribuição de medicamentos e 40,7\% nada modificariam na instituição ou não responderam. 
Tabela 6- Sugestões dos participantes do estudo acerca de modificações a serem realizadas nas instituições. (Ribeirão Preto, 2003)

\begin{tabular}{llcc}
\hline & Sugestões & $\mathbf{N}^{\mathbf{0}}$ & $\%$ \\
\hline \multirow{2}{*}{ Implantação } & Dose unitária & 18 & 15,9 \\
& Reserva de medicamento no setor & 9 & 8 \\
& Dose individual & 8 & 7,1 \\
& Informatização & 6 & 5,3 \\
& Farmácia-satélite & 4 & 3,5 \\
& Dose mista(unitária e individualizada) & 2 & 1,8 \\
& Central de diluição & 2 & 1,8 \\
Modificações & Atitudes tomadas com profissional & 7,1 \\
& Forma de prescrição médica & 3,5 \\
& Distribuição do medicamento & 4 & 3,5 \\
Não fariam modificações & Solicitação de material e medicamento & 4 & 1,8 \\
Não responderam & & 2 & 23 \\
\hline \multirow{2}{*}{ Total } & & 26 & 17,7 \\
\hline
\end{tabular}

A Tabela 7 indica a opinião dos profissionais acerca do sistema de medicação na instituição. Para $61,7 \%$ (66) dos participantes, o sistema de medicação estava adequado, sendo que $25,2 \%$ (27) des-

Tabela 7 - Opinião dos profissionais segundo o sistema de medicação de sua instituição. (Ribeirão Preto, 2003)

\begin{tabular}{|c|c|c|}
\hline Opinião & $\mathrm{N}^{\mathrm{o}}$ & $\%$ \\
\hline Sistema adequado & 39 & 36,5 \\
\hline Sistema adequado, mas com falhas & 27 & 25,2 \\
\hline Subtotal & 66 & 61,7 \\
\hline Sistema inadequado & 14 & 13,1 \\
\hline Não responderaml & 27 & 25,2 \\
\hline Total & 107 & 100 \\
\hline
\end{tabular}

\section{DISCUSSÃO}

Com relação às prescrições, predominou nessas instituições a prescrição manual $(74,8 \%)$, a qual é um dos fatores que predispõe à ocorrência do erro na medicação. Alguns estudos afirmam que o uso do computador na prescrição médica reduz a porcentagem de erros de medicação, já que as ordens estão estruturadas (incluindo dose e freqüência), são legíveis e podem ser identificadas. Com esse sistema podem ser obtidas informações relativas ao cliente como as alergias e interações medicamentosas ${ }^{(4,13)}$.

Observou-se que $50,4 \%$ das instituições possuíam o sistema de distribuição por dose individualizada e em $18,7 \%$ por dose unitária. AASHP declara que o sistema de distribuição por dose unitária é uma estratégia efetiva para reduzir a freqüência de erros na medicação ${ }^{(9)}$. tes o consideraram adequado, mas apresentando falhas e para $13,1 \%$ (14), o sistema de medicação estava inadequado.
O sistema de medicação nos hospitais e sua avaliação por um grupo de profissionais
A dose unitária possui várias vantagens em relação aos outros sistemas de distribuição como: diminuição na incidência de erros; economia de custos; aumento da segurança do profissional médico na garantia da utilização do medicamento certo, na dose certa e hora certa, segundo a prescrição médica e maior integração do farmacêutico com os outros profissionais da equipe de saúde ${ }^{(14)}$.

Segundo os participantes, 91\% das causas dos erros foram associadas ao profissional e não ao sistema em si. Porém, o erro não tem apenas uma causa (o profissional), mas deve ser considerado como uma falha do sistema e não somente dos indivíduos ${ }^{(5)}$.

Devido a isso, devem ser analisadas as etapas do processo de medicação e desconsiderar o profissional como a única causa do erro ${ }^{(15)}$.

Os participantes informaram que $88,9 \%$ das atitudes tomadas pela instituição frente ao erro na 
Silvia H. De Bortoli Cassiani Thalyta Cardoso A. Teixeira Simone Perufo Opitz Josilene Cristina Linhares medicação foram em relação ao próprio profissional. Alguns participantes informaram que essas atitudes eram uma forma de punição ou advertência devido ao erro. Dessa maneira, torna-se difícil buscar as causas do erro, pois os profissionais sentem medo de relatar o erro e preferem omiti-lo. Assim, as atitudes tomadas pelas instituições frente ao erro não devem ter esse caráter punitivo e os relatos dos erros de medicação não devem ser vistos com propósito punitivo, mas como dados que permitam o desenvolvimento de ações educacionais e administrativas ${ }^{(16)}$.

Dessa forma, os relatos dos erros devem ser anônimos e voluntários, e os profissionais devem ser motivados pela instituição a fazê-los ${ }^{(12)}$.

Dos participantes, $43 \%$ não apresentaram sugestões para modificações nas instituições que favoreceriam o seu trabalho e melhorariam o sistema de medicação do hospital.

Portanto, $61,7 \%$ dos participantes consideraram adequados os sistemas de medicação nos hospitais, mesmo sabendo que eles apresentavam falhas que propiciariam erros. Porém, um sistema adequado visando à segurança do cliente tem por objetivo reduzir os fatores que possibilitem a ocorrência de erros pelos profissionais, permitindo assim a detecção e prevenção dos $\operatorname{erros}^{(17)}$.

\section{REFERÊNCIAS}

(1) Gandhi T, Seger D, Bates DW. Identifyning drug safety issues: from research to practice. Int J Qual Health Care 2000; 12(1):69-76.

(2) Zangwill AB, Bolinger AM, Kanei RK. Reducing prescribing errors through a quiz program for medical residents. Am J Health-Syst Pharm 2000; 57(15):1396-7.

(3) Cassiani SHB. Erros na medicação: estratégias de prevenção. Rev Bras Enferm 2000; 53(3):424-30.

(4) Bates DW. Using information technology to reduce rates of medication errors in hospitals. BMJ 2000; 320(7237):788-91.

(5) Pepper GA. Errors in drug administration by nurses. Am J Health Syst Pharm 1995; 52(4):390-5.

(6) Frey B, Buettiker V, Hug MI, Waldvogel K, Gesller $\mathrm{P}$, Ghelfi D et al. Does critical incident reporting contribute to medication error prevention? Eur J Pediatr 2002; 161(11):594-9.

\section{CONSIDERAÇÕES FINAIS}

Neste estudo, a maioria das instituições não possuía prescrição eletrônica e sistema de distribuição de medicamentos por dose unitária, os quais são importantes estratégias na prevenção do erro na medicação.

Muitos participantes associaram as causas dos erros de medicação ao profissional, e isso demonstrou a persistência na cultura de responsabilizar somente o indivíduo (profissional) pelo erro, não se levando em conta as falhas do sistema de medicação da instituição. E persiste ainda a prática de punição, quando detectado o erro na medicação, através de relatos e reuniões, sem modificação substancial da causa que levou ao erro.

Além disso, vários profissionais não apresentaram sugestões para modificações no sistema de distribuição da sua instituição, o que favoreceria o seu trabalho e acarretaria numa melhor assistência ao cliente.

Assim, neste estudo, a maioria dos participantes considerou inadequado o sistema de medicação de sua instituição, mesmo sabendo que ele apresentava falhas que propiciariam o erro na medicação.

Dessa maneira, sugerimos novas investigações sobre a temática, visto que os erros são indicadores de qualidade na assistência ao cliente.

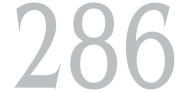

Rev Esc Enferm USP $2005 ; 39(3): 280-7$.
(7) Lesar TS, Lomaestro BM, Pohl H. Medicationprescribing errors in a teaching hospital. Arch Intern Med 1997; 157(14):1569-76.

(8) Leape LL, Cullen DJ, Clapp MD, Burdick E, Demonaco HJ, Erickson JI et al. Pharmacist participation on physician rounds and adverse drug events in the intensive care unit. JAMA 1999; 282(3):267-71.

(9) Top-priority actions for preventing adverse drug events in hospitals. Recommendations of an expert panel. Am J Health Syst Pharm 1996; 53(7): 747-51.

(10) Barker K, Flynn E, Pepper G, Bates DW, Mikeal RL. Medication error observed in 36 health care facilities. Arch Intern Med 2000; 12(16):1897903. 
(11) Souza LO, Carvalho APS, Chianca TCM, Freitas MEA, Ricaldon CAC. Classificação de erros de medicação ocorridos em um hospital privado de Belo Horizonte. REME: Rev Mineira Enferm 2000; 4:2-8.

(12) Stump LS. Re-engeneering the medication errorreporting process: removing the blame and improving the system. Am J Health Syst Pharm 2000; 57(suppl 4):S10-17.

(13) Kaushal R, Barker KW, Bates DW. How can information technology improve patiente safety and reduce medication errors in children's health care? Arch Pediatrs Adolesc Med 2000; 155(9):1002-7.

(14) Coimbra JAH, Valsechi EASS, Carvalho MDB, Pelloso SM. Sistema de distribuição de medicação por dose unitária: reflexões para a prática da enfermagem. Rev Lat-Am Enferm 1998; 6(4):15-9.
(15) Anderson DJ, Webster CS. A system approach to the reduction of medication error on the hospital ward. J Adv Nurs 2001; 35(1):34-41.

(16) Carvalho VT, Cassiani SHB. Erros de medicação e conseqüências para profissionais de enfermagem e clientes: um estudo exploratório. Rev Lat-Am Enferm 2002; 10(4):523-9.

(17) Leape LL, Bates DW, Cullen DJ, Cooper J, Demonaco HJ, Gallivan T et al. Systems analysis of adverse drug events. JAMA 1995; 274(1): 35-43.
O sistema de medicação nos hospitais e sua avaliação por um grupo de profissionais 